Rev. Saride puibl., S. Paulo

1E(supl.): 64-78, 1981

\title{
AVALIAÇÃO INICIAL DOS EFEITOS PSICO-PEDAGÓGICOS DOS CENTROS DE EDUCAÇÃO E ALIMENTAÇÃO DO PRÉ-ESCOLAR *
}

José Fernandes**

Yaro R. Gandra***

\begin{abstract}
FERNANDES, J. \& GANDRA, Y. R. Avaliação inicial dos efeitos psico-pedagógicos dos Centros de Educação e Alimentação do Pré-escolar. Rev. Saúde públ., S. Paulo, 15(supl.):64-78, 1981.
\end{abstract}

RESUMO: Foi avaliado o desenvolvimento sócio-psicomotor dos pré-escolares submetidos ao processo de recreação do Centro de Educação e Alimentação do Pré-escolar (CEAPE) quando comparados com um grupo controle da mesma comunidade, por meio de um Instrumento de Avaliação do Desenvolvimento do Pré-escolar (IADPE) organizado e pré-testado para este fim. Este instrumento mostrou-se sensivel para avaliar as diferenças obtidas. Os pré-escolares foram também avaliados por uma "Escala de Comportamento". Tanto em uma avaliação fechada (IADPE) como numa avaliação mais influenciada por fatores subjetivos ("Escala"), revelaram-se diferenças significativas em favor das crianças que frequentavam o programa ("Ceapenses") em relação aos que não frequientavam ("Não-Ceapenses"). Conjectura-se a provável ocorrência de dificuldades no processo de escolarização das crianças que não freqüentavam o programa.

UNITERMOS: Pré-escolares, avaliação. Criança, desenvolvimento. Programa CEAPE, avaliação psicométrica.

\section{INTRODUÇÃO}

Os anos pré-escolares têm sido caracterizados como um periodo de grande vulnerabilidade, tanto em termos de aspectos físicos, como psicológicos. De fato, de um modo geral, alguns dos casos mais graves de desnutrição infantil ocorrem na idade pré-escolar; além disso, torna-se cada vez mais patente a importância da estimulação ambiental neste período, não só para o desenvolvimento emocial, como também para o desenvolvimento de aspectos cognitivos, perceptivos, entre outros, que por sua vez, irão fornecer as bases pará outros eventos futuros, como a escolarização, por exemplo (Poppovic e col.10, 1975).

Estes dois fatores - alimentação adequada e estimulação ambiental de certos aspectos psicológicos nem sempre podem ser inteiramente satisfeitos pelo meio familiar em que o pré-escolar vive, especialmente nas camadas de baixo nivel sócio-econômico, que formam as porções mais expressivas de nossa população. Além disso, não há uma assistência suficiente ao pré-escolar

* Convênio 10/77 - INAN/DN/FSP/USP

** Do Programa CEAPE - Departamento de Nilrição da Faculdade de Saúde Pública da USP

*** Do Departamento de Nutrição da Faculdade de Saúde Pública da USP - At. Dr. Arnaldo, 715 - 01255 - São Paulo, SP - Brasil 
FERNANDES, J. \& GANDRA, Y.R. Avaliação inicial dos efeitos psico-pedagógicos dos Centros de Educação e Alimentação do Pré-Escolar. Rev. Saúde puibl., S. Paulo, 15(supl.): 64-78, 1981.

por parte dos serviços públicos existentes. Como conseqüência, pode-se observar, entre outras coisas, a ocorrência de altas taxas de evasão e repetência na primeira série do primeiro grau e os seus sérios efeitos, a nivel individual e social.

Em função da gravidade destes aspectos em nosso país, são necessárias medidas urgentes e viáveis que permitam dar alguma colaboração para a solução destes problemas. Uma das linhas de atuação, nesse sentido, é a implantação de programas preventivos de educaçăo escolar. O programa CEAPE - Centros de Educação e Alimentação do Pré-Escolar - (Gandra, 1973, 1976) ${ }^{5,6}$ desenvolvido atualmente no interior do Estado de São Paulo, é um modelo alternativo para $o$ atendimento ao pré-escolar de 2 a 6 anos de idade. Além da suplementação alimentar, o programa tem por objetivo a estimulação de aspectos cognitivos, motores, perceptivos, de linguagem, de expressão e conhecimentos sociais. Estes aspectos são abordados através de atividades psico-pedagógicas selecionadas na forma de recreação orientada (jogos, dramatizações, pintura, entre outras), que são executadas segundo sugestão oferecida pelo "Plano Rotativo de Recreação Orientada" 3. Em princípio, as atividades não são seqüenciadas e nem são divididas por faixa etária. A tarefa de "programação" das atividades sugeridas no Plano Rotativo cabe à professora.

A praticidade, flexibilidade e baixo custo do programa (mobilizaçāo de recursos ociosos da comunidade) tornaram factivel sua expansão através de várias cidades do interior do Estado de São Paulo, e a implantação de programas semelhantes na sua $\mathrm{Ca}$ pital (exemplo, Plano de Educação Infantil (PLANEDI)) e em outros Estados (exemplo: Programa de Assistência ao Pré-Escolar (PROAPE), em Pernambuco, CEAPE no Rio Grande do Sul). De um modo geral, cada comunidade implanta o programa de acordo com seus recursos disponiveis no momento, obedecendo, entretanto, às diretrizes gerais do programa: oferecimento de recreação orientada, de suplementação alimentar (merenda) e exigência de participação ativa da mãe do pré-escolar nas atividades desenvolvidas, permitindo-lhe "multiplicar" no lar os conhecimento adquiridos (Gandra, 1973) ${ }^{5}$.

Existem dados na literatura mostrando que atividades de recreação são menos eficazes, em termos de desenvolvimento cognitivo de pré-escolares, que exercícios cognitivos altamente estruturados e diretamente executados, sob a orientação de pessoal especializado. Entretanto, este tipo de estratégia, muito estruturada e sofisticada, exige alta soma de recursos humanos e materiais, o que não torna factivel a implantação destes programas educacionais em maior escala.

Dessa forma, torna-se importante a tarefa dos programas alternativos mais simples, como o CEAPE, pois eles são atualmente mais viáveis em termos de implantação a curto prazo e de atendimento a um grande número de crianças, conforme mostrou a expansão do programa CEAPE no Estado de São Paulo e de programas semelhantes, em outros Estados. Restava, pois salientar, se um programa, como o CEAPE, de tão baixa sofisticação era eficaz ou não em termos de promover progresso sócio-psico-pedagógico nos pré-escolares que o freqüentavam.

Muitos são os programas que incluem em seu bojo uma série de atividades psico-pedagógicas, mas poucos são os que avaliam a eficácia obtida. Quando o fazem, geralmente adotam sistemas alienigenos, sem ao menos adaptá-los às condições culturais locais.

A escolha de um sistema de avaliação do desenvolvimento infantil entretanto, não é tarefa fácil, dependerá, entre outras coisas, das características culturais do grupo sob estudo.

A própria sensibilidade de um simples teste pode variar de acordo com o grau de desenvolvimento que a criança apresentar. Um sistema de avaliação poderá ser sensivel quando aplicado a crianças de comunidades de nível sócio-econômico baixo e não o ser 
FERNANDES, J. \& GANDRA, Y.R. Avaliação inicial dos efeitos psico-pedagógicos dos Centros de Educação e Alimentação do Pré-Escolar. Fev. Saúde públ., S. Paulo, 15(supl.): 64-78, 1981.

para crianças das classes média ou alta. Por outro lado, a confirmação de mudança do estágio de desenvolvimento não significa sempre que ela tenha ocorrido como conseqüência do programa sócio-cultural oferecido. Interações advindas da própria comunidade e da familia poderão igualmente influenciar nos resultados da avaliação. Dificilmente pois, se poderá tirar conclusões definitivas se não se organizar um grupo controle retirado da mesma comunidade e com características semelhantes às do grupo em estudo. Nesta ordem de idéias é que se decidiu trabalhar sempre com um grupo de comparação.

Dentro desta ótica, o sistema de avaliação que teríamos que organizar deveria ser adequado à população a ser avaliada tanto quanto à idade, como ao padrão sócio-cultural. Deveria ser também de fácil aplicação, econômico, rápido e sensivel às variações comportamentais próprias da cultura em foco. Deveria ainda ser de tal simplicidade que agentes multiplicadores da ação, treinados e supervisionados por profissionais especializados, aumentariam a abrangência da avaliação em curto tempo. Desde o início não se esperava poder tirar conclusões individuais mas, somente para grupos de individuos. As conclusões individuais requerem métodos mais sofisticados, mais demorados e mais custosos e insubstituiveis.

O trabalho inicial de avaliação psico-pedagógica do programa CEAPE foi desenvolvido por Poppovic e Campos (1975) ${ }^{9}$, atendendo à solicitação feita pelo Departamento de Nutrição da Faculdade de Saúde Pública. Estas pesquisadoras escolheram o teste "Preschool Inventary" - (Caldwell, $1970)^{2}$ como ponto de partida, uma vez que este teste apresenta uma amostragem adequada de conteúdos, e seu material é barato, de aplicação individual breve $e$ razoavelmente simples. É uma das várias versões de testes concebidos por Caldwell ${ }^{2}$ para avaliar os efeitos educacionais do programa Head Start, em criançás norte-americanas culturalmente marginalizadas. As dife- rentes versões têm muitos pontos em comum; elas não se propõem a medir capacidades psicológicas básicas - como inteligência, por exemplo - mas sim, fornecem avaliações de certas habilidades ou desempenhos tidos como necessários para a escolarização posterior. O conteúdo do "Preschool Inventary" abrange essencialmente conceitos elementares de forma, cor, tamanho, posição, aspectos relacionados com contagem, conhecimento de partes do corpo e sobre o meio ambiente, execução de ordens e alguns aspectos de coordenação viso-motora. Nesse sentido, o "Preschool Inventary" pode ser considerado mais como um teste de aproveitamento educacional (Anastasi, 1967) ${ }^{1}$ do que como um teste propriamente psicológico, embora não se possa traçar uma distribuição rígida entre estes dois tipos de teste. De fato, o "Preschool Inventary" foi planejado de forma a ser aplicado pelas próprias professoras do Head Start, depois de um treinamento breve.

Apesar de ressalvas quanto à interpretação de determinados resultados dos testes de Caldwell ${ }^{2}$, o conteúdo, o modo simples de aplicação e correção do "Preschool Inventary" foram considerados bastante promissores para o uso de um programa como - CEAPE. Com base nestes ponto, Poppovic e Campos $(1975)^{9}$ realizaram a adaptação inicial deste teste retirando itens considerados inadequados para a nossa população em estudo. Paralelamente também foi acrescentada uma versão resumida da "Escala de Comportamento Intra-Teste", utilizada para avaliar o comportamento da criança durante o teste. A adaptação de itens foi denominada "Inventário de Habilidades para Pré-Escolares" e testada em outubro de 1975, com o objetivo básico de avaliar o grau de discriminação deste instrumento entre as idades de 4,5 e 6 anos. Os resultados obtidos foram bastante encorajadores e apontaram para os benefícios de um trabalho mais intensivo com o "Inventário".

Com o propósito de aumentar a sensibilidade do instrumento original, o comportamento de cada item de per si, foi analisado, 
FERNANDES, J. \& GANDRA, Y.R. Avaliação inicial dos efeitos psico-pedagógicos dos Centros de Educação e Alimentação do Pré-Escolar. Rev. Saúde públ., S. Paulo, 15(supl.): 64-78, 1981.

eliminando-se aqueles que não conseguiram alto grau de discernimento entre os pré-escolares dos diferentes grupos etários. Posteriormente, foram ainda adicionados outros itens perfazendo um total de 70 .

A versão inicial do teste sofreu desta forma modificações originando o "Instrumento de Avaliação do Desenvolvimento de Pré-Escolares (IADPE)" com 70 itens. Novamente, avaliou-se o grau de discriminação deste instrumento entre crianças "Ceapenses" e "Não-Ceapenses" de 4, 5 e 6 anos de idade e de baixo nivel sócio-econômico, da cidade de Leme (SP). Analisou-se também uma série de características estatísticas deste teste e da "Escala de Comportamento" *.

Os resultados obtidos indicaram que o "IADPE" e a "Escala" discriminavam significativamente entre as idades de 4,5 e 6 anos, tanto para crianças "Ceapenses" como para "Não-Ceapenses". O "IADPE" mostrou-se bastante preciso (coeficiente de precisão KR-20 entre 0,85 e 0,92 ), e com erros padrões de medida razoavelmente estáveis (entre 3,0 e 3,5 pontos).

Estes resultados, analisados juntamente com a seleção do conteúdo dos itens do "IADPE", mostraram a adequação deste teste como um instrumento útil de avaliação psico-pedagógica de pré-escolares, de 4 a 6 anos, de baixo nível sócio-econômico.

Em função destes dados, o objetivo do presente trabalho foi o de comparar os desempenhos de crianças "Ceapenses" e "Não-Ceapenses" no "IADPE" e nas avaliações obtidas através da "Escala de Comportamento".

\section{METODO}

\section{Seleção e Descrição dos CEAPEs Utilizados}

Conforme já foi aventado, o programa CEAPE expandiu-se por diversas cidades do interior do Estado de São Paulo. Devido às peculiaridades de cada comunidade, esta expansão gerou, em termos funcionais, certo grau de variabilidade entre os diferentes
CEAPEs, embora todos seguissem as linhas básicas gerais do programa. Em função destes pontos, optou-se por centralizar os trabalhos de avaliação numa cidade que possuisse um número razoável de CEAPEs, operando sob uma orientação comum. Escolheu-se a cidade de Leme, onde no início das atividades de pesquisa havia cerca de 12 CEAPEs, cada um atendendo de 30 a 40 crianças, e funcionando sob supervisão educacional direta da Prefeitura local.

Em Leme, todos os CEAPEs funcionam em locais fechados e cobertos, durante cerca de 3 a 4 horas diárias. Embora a admissão aos CEAPEs seja aberta a qualquer pré-escolar, a população visada pelo programa é essencialmente a de baixo nível sócio-econômico, residente nas proximidades cos centros. No período da manhã, são atendidas preferencialmerite as crianças de 6 anos e, à tarde, as menores. Todas as crianças recebem suplementação alimentar (merenda) diariamente.

No nível educacional, as atividades do Plano Rotativo de Recreação Orientada: caracterizam o atendimento às crianças (jogos, dramatizações, canto, recorte, pintura, entre outras). Estas atividades visam o desenvolvimento de aspectos cognitivos, motores, perceptivos, de linguagem, de expressão e conhecimento sociais. As crianças de 6 anos, além destas atividades, recebem cutras mais claramente ligadas à escolarização, como exercícios de contagem, cópia ae traços, entre outros.

O trabalho junto às crianças é realizado por professoras primárias, designadas pela municipalidade. As mães dos pré-escolares também participam ativamente no programa, exercendo atividades relacionadas com a merenda e com a recreação, sob orientação da professora.

\section{Caracteristicas da Amostra}

\section{a) Critérios de formação de grupos}

O "IADPE" e a "Escala", foram aplicados em crianças de 4,5 e 6 anos, resi- 
FERNANDES, J. \& GANDRA, Y.R. Avaliação inicial dos efeitos psico-pedagógicos dos Centros de Educação e Alimentação do Pré-Escolar. Rev. Saúde públ., S. Paulo, 15(supl.): 64-78, 1981.

dentes na cidade de Leme. Para cada idade, foram formados dois grupos: um de crianças que freqüentavam o programa CEAPE, "Ceapenses", e outro, por crianças "Não-Ceapenses".

As crianças "Ceapenses" inscreveram-se e freqüentaram o programa voluntariamente. Testadas, foram selecionadas com base na idade cronológica e no tempo de freqüência ao programa (no mínimo 6 meses letivos seguidos, com pelo menos, $75 \%$ de presença).

As crianças "Não-Ceapenses" foram sorteadas com base em um cadastramento de famílias de baixo nivel sócio-econômico, residentes nas "áreas de influência" de cada CEAPE de Leme. Definiu-se como "área de influência" a região da cidade de Leme, em torno de cada CEAPE, delimitada pelos endereços mais periféricos das famílias que tivessem crianças freqüentando aquele centro, a ocasião da pesquisa ("Ceapenses").

Todas as crianças "Não-Ceapenses", na ccasião da pesquisa, não freqüentavam (segundo os responsáveis pela criança), qualquer tipo de creche, pré-escola ou programas afins.

\section{b) Descrição dos grupos}

Foram testadas 353 crianças "Ceapenses" e 214 "Não-Ceapenses", com idades de 4, 5 e 6 anos. A frequiência média das crianças "Ceapenses" ao programa foi, no mínimo, de 6 meses letivos.

A Tabela 1 apresenta o número de crianças de cada grupo, por idade. Pode-se notar que as crianças "Ceapenses" não estão igualmente balanceadas pelas três idades. O grupo "Ceapense" de 6 anos é o mais numeroso e evidência a tendência real encontrada nos CEAPEs de Leme para o atendimento prioritário às crianças prestes a iniciar a primeira série do primeiro grau.

A Tabela 2 mostra as médias, os desvios-padrões e as medianas das idades, em meses, para cada grupo.

T A B E I A 1

Grupos de pré-escolares "ceapenses" e "não-ceapenses" distribuídos por idade. residentes na cidade de Leme, SP

\begin{tabular}{lccc}
\hline Idade & "Ceapenses" & "Não-ceapenses" & Total \\
\hline 4 anos & 42 & 77 & 119 \\
5 anos & 109 & 74 & $18 \%$ \\
6 anos & 202 & 63 & 265 \\
\hline Tot.l1 & 353 & 214 & 567 \\
\hline
\end{tabular}

T A B E L A 2

Médias, desvios-padrões (amostrais) e medianas das idades dos pré-escolares "ceapenses" e "não-ceapenses" residentes na cidade de Leme, SP.

\begin{tabular}{lcccccc}
\hline \multirow{2}{*}{ Idade } & \multicolumn{2}{c}{ "Ceapenses" } & \multicolumn{3}{c}{ "Não-ceapenses" } \\
\cline { 2 - 7 } & $\mathrm{X}(\mathrm{m})$ & $\mathrm{S}$ & $\mathrm{Md}$ & $\mathrm{X}(\mathrm{m})$ & $\mathrm{S}$ & $\mathrm{Md}(\mathrm{m})$ \\
\hline $\mathbf{4}$ anos & 55 & 3.07 & 56 & 55 & 3,45 & 55 \\
$\mathbf{5}$ anos & 66 & 3.48 & 66 & 65 & 3,40 & 66 \\
6 anos & 77 & 3,55 & 77 & 78 & 3,31 & 78 \\
\hline
\end{tabular}


FERNANDES, J, \& GANDRA, Y.R. Avaliação inicial dos efeitos psico-pedagógicos dos Centros đe Educação e Alimentação do Pré-Escolar. Rev. Saúde públ., S. Paulo, 15(supl.): 64-78, 1981.

As Tabelas 3 a 6 referem-se a dados que foram coletados em entrevista, com a mãe ou responsável pela criança.

Os dados de uma mesma família foram computados apenas uma vez. Dessa forma, o número de familias é menor que o número de crianças testadas, dada a existência de irmãos entre elas.
A Tabela 3 apresenta o tamanho da Família Nuclear das crianças "Ceapenses" e "Não-Ceapenses". A familias de crianças "Não-Ceapenses" tenderam, em termos relativos, a ser maiores que as famílias de crianças "Ceapenses".

A Tabela 4 mostra a renda familiar mensal per capita (bruta), em termos de salários mínimos.

T A B E L A 3

Tamanho da família nuclear (declarado em entrevista), para cada grupo de pré-escolares "ceapenses" e "nāo-ceapenses" residentes na cidade de Leme. SP.

\begin{tabular}{|c|c|c|}
\hline Tamanho & "Ceapenses" & "Näo-ceapenses" \\
\hline $2 \mid-14$ & $122(37,89 \%)$ & $46(25,84 \%)$ \\
\hline $5|-| 6$ & $126(39,13 \%)$ & $68(38,20 \%)$ \\
\hline $7-1-8$ & $46(14,29 \%)$ & $43(24,16 \%)$ \\
\hline $9 \mid-10$ & $20(6.21 \%)$ & $14(7,87 \%)$ \\
\hline 11 ou $t$ & $8(2,48 \%)$ & $7(3.93 \%)$ \\
\hline Total & 322 & 178 \\
\hline Sem informação & 17 & 10 \\
\hline
\end{tabular}

T A B E L A 4

Renda familiar mensal per capita em termos de salários minimos, dos grupos de pré-escolares "ceapenses" e "não-ceapenses", residentes na cidade de Leme, SP.

\begin{tabular}{|c|c|c|c|c|}
\hline \multirow{2}{*}{$\begin{array}{c}\text { Renda familiar } \\
\text { per capita }\end{array}$} & \multicolumn{2}{|c|}{ "Ceapenses" } & \multicolumn{2}{|c|}{ "Não-ceapenses" } \\
\hline & No & $\%$ & No & $\%$ \\
\hline $0-0,5$ sal. mín. & 126 & 40,00 & 141 & 83,43 \\
\hline $0,5 \longrightarrow 1,0$ sal. mín. & 154 & 48,89 & 24 & 14,20 \\
\hline $1.0-1,5$ sal. min. & 25 & 7,94 & 4 & 2,37 \\
\hline 1,5 ou + sal. mín. & 10 & 3,18 & 0 & - \\
\hline Total & 315 & & 169 & \\
\hline Sem informação & 24 & & 19 & \\
\hline
\end{tabular}


FERNANDES, J. \& GANDRA, Y.R. Avaliação inicial dos efeitos psico-pedagógicos dos Centros de Educação e Alimentação do Pré-Escolar. Rev. Saúde públ., S. Paulo, 15(supl.): 64-78, 1981.

Nota-se que em ambos os grupos a grande maioria das famílias apresentou renda familiar per capita menor que um salário mínimo.

A Tabela 5 mostra o nivel de escolaridade dos pais e mães das crianças testadas.

A Tabela 6 mostra o nivel de prestígio ocupacional do chefe da família, segundo a Escala de Hutchinson, modificada por Gouveia $(1970)^{i}$. As profissões foram classificadas de acordo com os critérios de Macedo (1973) ${ }^{8}$. Em ambos os grupos houve uma concentração nos níveis mais baixos da Escala de prestígio ocupacional.

Para resumir os dados levantados até agora, pode-se dizer que ambos os grupos de crianças - "Ceapense" e "Não-Cea-
Fense" - foram originários, no geral, de familias de baixo nível sócio-econômico, em termos de renda, escolaridade dos pais e nivel de prestígio ocupacional do chefe da família. É importante assinalar que esta caracterização sócio-econômica está influenciada pela relatividade inerente ao método utilizado para a coleta de dados. Apesar disso, eles aparentemente servem como uma caracterização, ainda que grosseira, dos grupos estudados.

Caracteristicas dos Instrumentos de Avaliação do Desenvolvimento do Pré-Escolar $(I A D P E)^{*}$

O "IADPE" é um teste de aproveitamento (achievement test), cujo objetivo é avaliar alguns aspectos elementares, consi-

T A B E L A 5

Escolaridade de pais e mães dos pré-escolares "ceapenses" e "não-ceapenses" residentes na cidade de Leme, $\mathbf{S P}$

\begin{tabular}{|c|c|c|c|c|}
\hline \multirow{2}{*}{ Escolarilade * } & \multicolumn{2}{|c|}{ "Ceapenses" } & \multicolumn{2}{|c|}{ "Não-ceapenses" } \\
\hline & Pais & Mães & Pais & Mães \\
\hline Sem instrução & $25(8.22 \%)$ & $32(10,26 \%)$ & $35(21,34 \%)$ & $58(33,53 \%)$ \\
\hline $\begin{array}{l}\text { Primário incompleto } \\
\text { (1-3 anos) }\end{array}$ & $120(39,47 \%)$ & $137(43,91 \%)$ & $88(53,66 \%)$ & $77(44,51 \%)$ \\
\hline $\begin{array}{l}\text { Primário completo } \\
\qquad(4-5 \text { anos })\end{array}$ & $139(45,72 \%)$ & $130(41,67 \%)$ & $37(22,56 \%)$ & $34(19,65 \%)$ \\
\hline $\begin{array}{l}\text { Ginásio incompleto } \\
\qquad(6-7 \text { anos })\end{array}$ & $6(1,97 \%)$ & $7(2,24 \%)$ & $2(1,22 \%)$ & $4(2.31 \%)$ \\
\hline $\begin{array}{l}\text { Ginásio completo } \\
(8-9 \text { anos })\end{array}$ & $6(1,97 \%)$ & $4(1,28 \%)$ & $2(1.22 \%)$ & 0 \\
\hline $\begin{array}{l}\text { Colegial ou }+ \\
(10 \text { anos ou }+)\end{array}$ & $8(2,63 \%)$ & $2(0,64 \%)$ & 0 & 0 \\
\hline Total & 304 & 312 & 164 & 173 \\
\hline Sem informação & 35 & 27 & 24 & 15 \\
\hline
\end{tabular}

* Adotou-se a nomenclatura vigente na ocasião em que os entrevistados eram escolares.

- O Manual de Instruções e o material do "IADPE" podem ser encontrados junto ao Departamento de Nutrição da Faculdade de Saúde Pública - USP. As características estatísticas do "TADPE" e "Escala" foram discutidas e publicadas em bibliografia citada 4. 
FERNANDES, J. \& GANDRA, Y.R. Avaliação injcial dos efeitos psico-pedagógicos dos Centros de Educação e Alimentação do Pré-Escolar. Rev. Saúde públ., S. Paulo, 15(supl.): 64-78, 1981.

derados básicos para a escolarização. A análise das características estatísticas principais deste instrumento consta em outro trabalho'. Todos os itens admitem apenas as respostas "certo" (valor 1) ou "errado" (valor zero). Estes valores são somados e constituem o "Total do Instrumento" cujo máximo é 70 pontos ${ }^{4}$.

A aplicação do "IADPE" é individual, leva cerca de $25 \mathrm{~min}$ por criança e o sell material é formado por elementos concretos simples, facilmente encontrados na comunidade. Também foi utilizada a "Escala de Comportamento Intra-Teste", cujo objetivo é avaliar o comportamento da criança durante o teste, com relação ao examinador e à tarefa. Esta "Escala" inclui 6 itens: quantidade de fala, persistência, atenção, auto-confiança, "rapport" e participação durante o teste. Cada item é avaliado pelo examinador, após a aplicação do "IADPE", numa escala de 1 a 5 pontos (discretos), definidos operacionalmente. O ponto 1 corresponde a pior realização no item, e o ponto 5 a melhor realização. Estes valores são somados e constituem o "Total da Escala”, cujo máximo é 30 pontos.

\section{T A B E L A 6}

Nivel de prestígio ocupacional do chefe da familia * de pré-escolares "ceapenses" e "nào-ceapenses" residentes na cidade de Leme. SP.

\begin{tabular}{|c|c|c|}
\hline Nivel de prestigio ocupacional & "Ceapenses" & "Não-ceapenses" \\
\hline Ocupações manuais não especializadas & $124(40,52 \%)$ & $109(66,46 \%)$ \\
\hline $\begin{array}{l}\text { Ocupações manuais especializadas e seme- } \\
\text { lhantes }\end{array}$ & $150(49.02 \%)$ & $48(29.27 \%)$ \\
\hline $\begin{array}{l}\text { Supervisão de trabalho manual e seme- } \\
\text { lhantes }\end{array}$ & $3(0,98 \%)$ & 0 \\
\hline $\begin{array}{l}\text { Ocupações não manuais de rotina e seme- } \\
\text { lhantes }\end{array}$ & $10(3,27 \%)$ & 0 \\
\hline $\begin{array}{l}\text { Proprietários de pequenas empresas co- } \\
\text { merciais e semelhantes }\end{array}$ & $13(4,25 \%)$ & $1(0,61) \%$ \\
\hline Aposentados & $6(1,96 \%)$ & $6(3,66 \%)$ \\
\hline Total & 306 & 164 \\
\hline Sem informação & 33 & 24 \\
\hline
\end{tabular}

\section{Procedimento}

Todas as crianças foram avaliadas com o "IADPE" e com a "Escala" nas instalações dos CEAPEs da cidade de Leme, por uma equipe formada por psicólogos ou estudantes dos últimos anos do Curso de Graduação do Instituto de Psicologia da Universidade de São Paulo. Todos os elementos da equipe foram previamente treinados quanto ao uso e avaliação do material, sendo também supervisionados durante todo trabalho de campo

\section{RESULTADOS}

Os dois grupos - "Ceapense" e "Nãu-Ceapense" - serăo comparados, idade por idade, com relação ao total do "IADPE" dos agrupamentos de itens do "IADPE" e do total da "Escala de Comportamento". 
FE'RNANDES, J. \& GANDRA, Y.R. Avaliação inicial dos efeitos psico-pedagógicos dos Centros de Educação e Alimentação do Pré-Escolar. Rev. Saúde públ., s. Paulo, 15(supl.): 64-78, 1981.

\section{Totais dos resultados do "IADPE"}

A Figura 1 apresenta os resultados obtidos com o "IADPE" em cada grupo e idade, na forma de medianas e quartis.

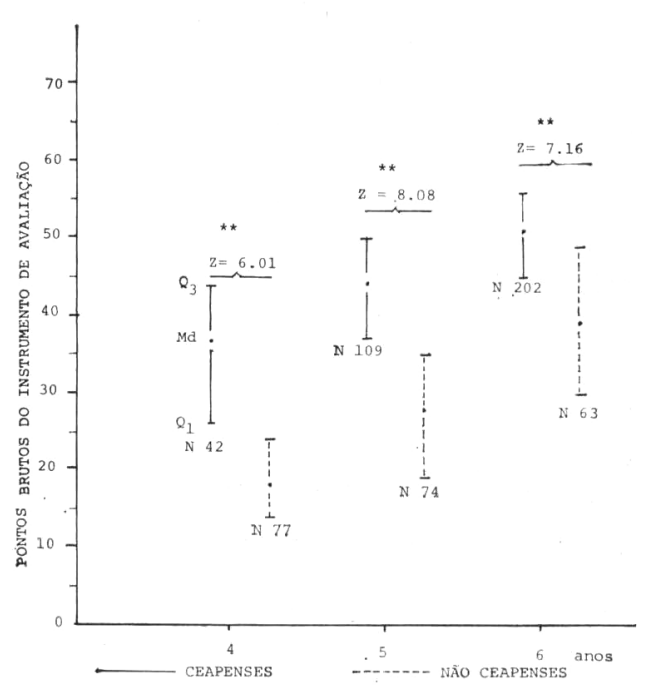

Fig. 1 - Medianas e quartis do total do "IADPE" aplicado em pré-escolares "ceapenses" e "não-ceapenses" residentes na cidade de Leme, SP.

Diferenças significantes. com $p<$ 001 por Mann-Whitney.
Pode-se observar que as crianças "Ceapenses" obtiveram resultados consistentemente mais elevados que as crianças "Não-Ceapenses", de mesma idade. Em ambos os grupos, pode-se observar uma tendência crescente praticamente linear, em função da idade, em termos de resultados medianos. As diferenças entre os valores medianos de grupos da mesma idade vão progressivamente diminuindo, dos 4 aos 6 anos. Esta diminuição era esperada uma vez que se utilizou sempre o mesmo instrumento para todos os grupos etários em benefício da simplificação desejada na avaliação do programa em nível de campo.

Conforme mostra a Tabela 7 , esta tendência à diminuição das diferenças pode ser também observada, em termos das médjas aritméticas dos resultados. Mesmo assim verifica-se que somente aos 6 anos " grupo "Não-Ceapense" estudado atingiu um resultado mediano semelhante ao obtido pelo grupo "Ceapense", já aos 4 anos.

As diferenças entre os resultados de grupos da mesma idade foram testadas através da prova não-paramétrica de Mann-Whitney (Siegel 11, 1975). Conforme mostra a Tabela 7 , para todas as idades foram encontradas diferenças altamente significativas $(p<001)$ entre os grupos "Ceapense" e "Não-Ceapense".

T A B E L A 7

Médias, desvios-padrões (amostrais) e medianas do total do "IADPE" aplicado em pré-escolares "ceapenses" e "não-ceapenses" residentes em Leme, SP.

\begin{tabular}{llll}
\hline Idade & Medidas & "Ceapenses" & "Não-ceapenses" * \\
\hline \multirow{3}{*}{4 anos } & $\mathrm{X}$ & 35,5 & 20,8 \\
& $\mathrm{~S}$ & 11,68 & $9,65 \quad(\mathrm{Z}=6,01 ;<001)$ \\
\hline \multirow{3}{*}{5 anos } & $\mathrm{Md}$ & 36,5 & $\mathbf{1 8 , 4}(\mathrm{Z}=\mathbf{2 8 , 2}$ \\
& $\mathrm{X}$ & 43,6 & $\mathbf{1 1 , 2 9}$ \\
& $\mathrm{S}$ & 8,91 & $27,3 \quad(\mathrm{Z}=8,08 ;<001)$ \\
\hline \multirow{3}{*}{ anos } & $\mathrm{Md}$ & 43,8 & 38,4 \\
& $\mathrm{X}$ & $\mathbf{5 0 , 5}$ & $11,23 \quad(\mathrm{Z}=7,16 ;<001)$ \\
\hline
\end{tabular}

* Entre parênteses ( ) encontram-se os valores $Z$ obtidos por meio da prova não paramétrica de Mann-Whitney (Siegel, 1975) 11. 
FE:RNANDES, J. \& GANDRA, Y.R. Avaliação inicial dos efeitos psico-pedagógicos dos Centros de Educação e Alimentação do Pré-Escolar. Rev. Saúde públ., S. Paulo, 15(supl.): 64-78, 1981.

\section{Agrupamentos de Itens do "IADPE"}

A fim de investigar mais detalhadamente as diferenças entre os grupos, foram definidos 10 agrupamentos mutuamente exclusivos, com os itens do Instrumento de Avaliação.

A tarefa de partição do "IADPE" em agrupamentos mutuamente exclusivos apresentou algumas dificuldades, já que vários itens avaliam, ao mesmo tempo, mais de um aspecto. Dessa forma, as classificações de itens em agrupamentos envolveram, em alguns casos, critérios arbitrários. A orientação geral seguida foi reunir itens que avaliem predominantemente caracteristicas em comum.
A Tabela 8 apresenta os agrupamentos definidos, o total máximo de pontos do agrupamento e as características avaliadas.

As médias de pontos, em cada agrupamento, foram transformadas em percentagens de acerto, com relação ao total máximo de pontos do agrupamento, através da expressão:

Média de pontos obtida

Total máximo do agrupamento

Esta transformação facilita a comparação entre agrupamentos de totais máximos diferentes.

A Figura 2 apresenta as médias de pontos obtidas em cada agrupamento, expressas em percentagens do seu total máximo, para cada idade em cada grupo.

T A B E L A 8

Agrupamentos de itens do "IADPE" utilizados e suas caracterizações.

\begin{tabular}{|c|c|c|}
\hline Agrupamentos & $\begin{array}{c}\text { Total } \\
\text { máximo } \\
\text { de pontos }\end{array}$ & $\begin{array}{l}\text { Tarefas pedidas } \\
\text { Aspectos avaliados }\end{array}$ \\
\hline I. Cópia & 4 & $\begin{array}{l}\text { cópia de figuras geométricas simples; (coordenação } \\
\text { viso-motora) }\end{array}$ \\
\hline II. Equilíbrio & & manutenção do equilibrio corporal estático e dinâmico \\
\hline III. Memória & 5 & $\begin{array}{l}\text { imitação de movimentos rápidos (atenção e memória } \\
\text { de curta duração) }\end{array}$ \\
\hline IV. Corpo & 6 & nomeação c reconhecimento de partes do corpo \\
\hline V. Cor & 4 & reconhecimento de cores básicas \\
\hline VI. Geometria & 4 & reconhecimento de figuras geométricas simples \\
\hline VII. Posição & 6 & utilizaçāo de conceitos espaciais \\
\hline VIII. Classificação & 8 & $\begin{array}{l}\text { classificação; estabelecimento de relações de iriclusão, } \\
\text { exclusão e oposição }\end{array}$ \\
\hline IX. Ordem & 16 & $\begin{array}{l}\text { comparação entre } 2 \text { elementos, com relação à } \\
\text { quantidade, tamanho, tempo e outros aspectos; } \\
\text { estabelecimento de relações de ordem entre mais de } 2 \\
\text { objetos; } \\
\text { seriação de } 5 \text { objetos }\end{array}$ \\
\hline X. Numeração & 7 & quantificação e aspectos relacionados \\
\hline
\end{tabular}



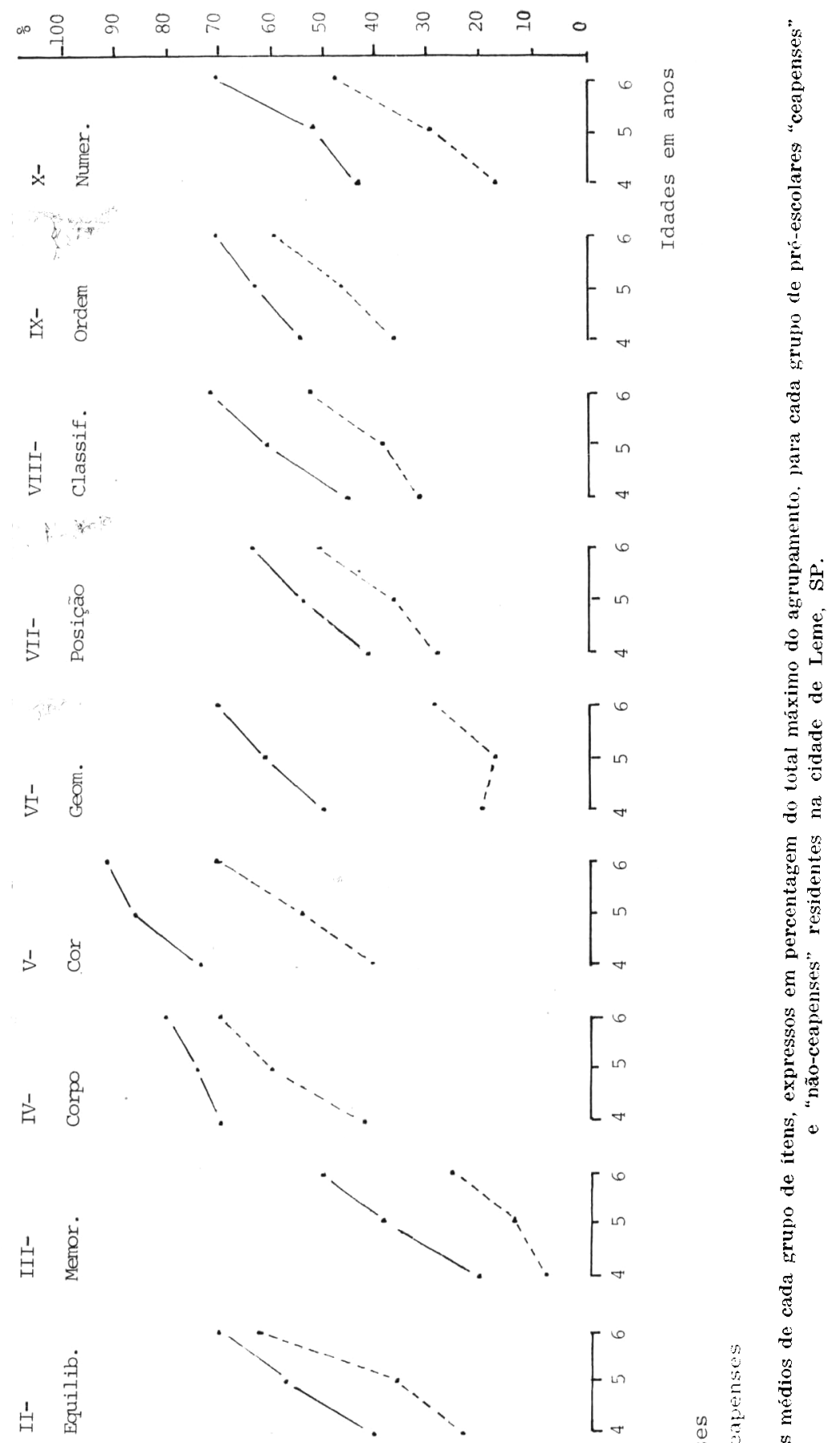
FERNANDES, J. \& GANDRA, Y.R. Avaliação inicial dos efeitos psico-pedagógicos dos Centros de Educação e Alimentação do Pré-Escolar. Rev. Saúde pủbl, S. Paulo, 15(supl.): 64-78, 1981.

Pode-se notar que todos os agrupamentos, conı exceção do VI-Geometria, para crianças "Não-Ceapenses", apresentavam médias de pontos crescentes, em função do aumento da idade. Além disso, comparando-se grupos de mesma idade, pode-se notar que o grupo "Ceapense" obteve sistematicamente resultados mais elevados que o "Não-Ceapense", em todos os agrupamentos. Para os "Ceapenses", os agrupamentos de maior facilidade (pelo menos $75 \%$ de acerto, em pelo menos duas idades consecutivas) foram: I - Cópia; IV - Corpo; V - Cor.

Nenhum agrupamento atingiu os critério de "facilidade", expostos acima, no grup. "Não-Ceapense". Isto indica que o "IADPE foi um teste difícil para este grupo, mesm aos 6 anos. Na maioria dos agrupamentos o grupo "Não-Ceapense" apresentou, aos anos, percentagens de acerto semelhante às do grupo "Ceapense", aos 4 ou 5 anos Estes resultados mostram, portanto, que a diferenças observadas entre os grupos, $n$ total do Instrumento não são atribuíveis diferenças existentes entre apenas algun agrupamentos de itens específicos, mas $\sin$ refletiram diferenças de desempenho a longo de todo o teste. De fato, em algur. agrupamentos, o resultado médio das criar ças "Não-Ceapenses" aos 6 anos é incl sive inferior ao das crianças "Ceapenses de 4 anos (I - Cópia; V - Cor; Vl Geometria).

Para o grupo "Ceapense", houve tendência para ganhos maiores na faixa de 4 - 5 anos do que na faixa de 5 - 6 anos, enl diversos agrupamentos. Por outro lado, no grupo "Não-Ceapense", existiu tendência a ganhos maiores na faixa de 5 - 6 anos do que na de 4 - 5 anos.

Com base nestes pontos, pode-se observar então que as diferenças entre os grupos, en termos de percentagens de acerto, são menores aos 6 anos que nas demais idades em vários agrupamentos. Apesar disso, como já foi levantado, apenas aos 6 anos o grupo "Não-Ceapense" apresentou, nestes agrupamentos, resultados equivalentes aos dos grupos "Ceapenses" de 4 ou 5 anos. Em alguns agrupamentos, entretanto, houve uma tendência a manter ou inclusive aumentar as diferenças entre os grupos, ao longo das idades (VI - Geometria; X - Numeração; III - Memorização; VIII - Classificação).

\section{Total da "Escala de Comportamento"}

A Figura 3 apresenta as medianas e quartis do total da "Escala de Comportamento", para cada idade dos grupos estudados.

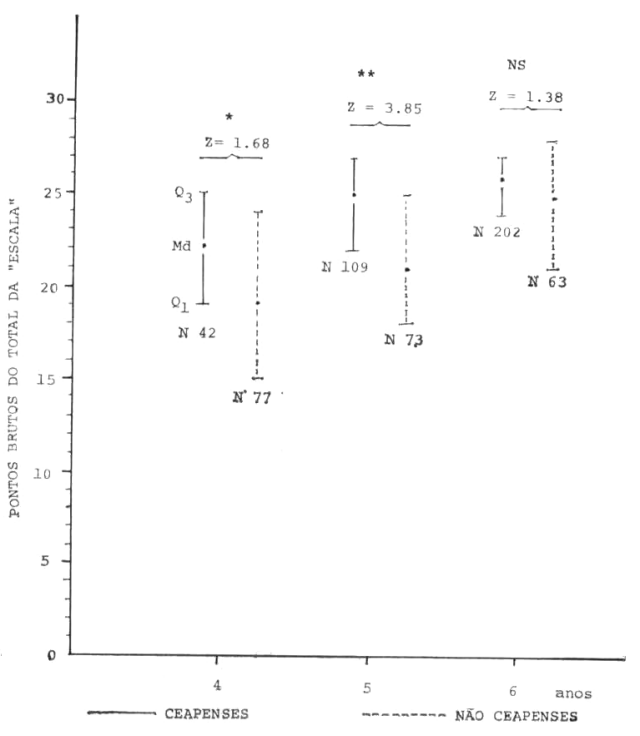

Fig. 3 - Medianas e quartis do total da "escala de comportamento intra-teste" aplicada em pré-escolares "ceapenses" e "não-ceapenses" residentes na cidade de Leme, SP.

** Diferenças com $\mathrm{p}<.001$; * diferenças com $\mathrm{p}<.05$ Mann-Whitney.

$\mathrm{NS}=$ Não significante.

Noramente observa-se, en ambos os grupos, tendência crescente nos resultados medianos, em função da idade. Conforme mostra a Tabela 9 , esta tendência também ocorre em termos de resultados médios.

As diferenças entre grupos de uma mesma idade foram testadas através da prova não-paramétrica de Mann-Whitney (Siegel ${ }^{11}$, 
FERNANDES, J. \& GANDRA, Y.R. Avaliação inicial dos efeitos psico-pedagógicos dos Centros de Educação e Alimentaçào do Pré-Escolar. Kev. Saúde pübl., S. Paulo, 15(supl.): 64-78, 1981.

1975). As diferenças entre os grupos deixaram de ser significativas aos 6 anos, conforme mostra a Tabela 9. Isto provavelmente aconteceu em função do "efeito de limite" da Escala, ou seja, devido a concentração de resultados nos valores muito próximos ao máximo do instrumento, com o aumento da idade. Novamente, observa-se que os maiores ganhos, em termos de medianos, ocorreram na faixa de $4-5$ anos para - grupo "Ceapense" e na de 5 - 6 anos, para o grupo "Nāo-Ceapense".

T A B E L A 9

Médias, desvios-padrões (amostrais) e medianas do total da "Escala de Comportamento Intra-teste" aplicada em pré-escolares "ceapenses" e "não-ceapenses" residentes em Leme, SP.

\begin{tabular}{|c|c|c|c|}
\hline Idade & Medidas & "Ceapenses" & "Não-ceapenses" * \\
\hline 4 anos & $\begin{array}{l}\mathrm{X} \\
\mathrm{S} \\
\mathrm{Md}\end{array}$ & $\begin{array}{c}20.9 \\
5,39 \\
22.0\end{array}$ & $\begin{array}{c}19.5 \\
5.57 \\
18.2 \quad(z=1,68 ; p<.05)\end{array}$ \\
\hline 5 anos & $\begin{array}{l}\mathrm{x} \\
\mathrm{s} \\
\mathrm{M} d\end{array}$ & $\begin{array}{c}24,0 \\
4.17 \\
24.6\end{array}$ & $\begin{array}{l}21.4 \\
4.54 \\
21.1 \quad(\mathrm{Z}=3,85 ; \mathrm{p}<.001)\end{array}$ \\
\hline 6 anos & $\begin{array}{l}\mathrm{X} \\
\mathrm{s} \\
\mathrm{Md}\end{array}$ & $\begin{array}{c}25.1 \\
3,41 \\
25,8\end{array}$ & $\begin{array}{l}24,0 \\
4,52 \\
25,0 \quad(Z=1,38 ; \text { N.S. })\end{array}$ \\
\hline
\end{tabular}

* Entre parenteses ( ) encontram-se os valores $Z$ obtidos por meio da prova não paramétrica de Mann-Whitney (Siegel, 1975)11.

\section{DISCUSSÃO E CONCLUSÓES}

Os resultados obtidos, em termos do total do "IADPE" permitiram constatar diferenças altamente significativas entre o grupo de crianças "Ceapenses" e "Não-Ceapenses". Embora as diferenças, em termos de valores medianos, tendam a diminuir ao longo das idades (aparentemente em função de se ter utilizado o mesmo instrumento para as diferentes faixas etárias), observou-se, aos 6 anos, uma defasagem de cerca de 2 anos entre os grupos, no total do Instrumento usado. Analisando-se mais detalhadamente as diferenças entre os grupos, ao longo de diversos agrupamentos de itens, observou-se a mesma tendência revelada pelo "total". Novamente, aos 6 anos, foram localizadas defasagens de 1 a 2 anos, entre os grupos "Ceapense" e "Não-Ceapense". As maiores diferenças entre os grupos surgiram em itens de cópia, de reconhecimento de cores e de figuras geométricas e de contagem numérica. Portanto, é licito supor que estas e outras diferenças sejam atribuíveis, pelo menos em algum grau, às atividades desenvolvidas pelo programa. A metodologia utilizada não permite avaliar de um modo inequivoco, a importância relativa de outros fatores alheios ao programa em si.

É importante assinalar que também foram observadas diferenças em aspectos mais básicos de atenção, memória a curto prazo e das operações cognitivas de classificação, seriação, entre outras. Enquanto que os primeiros aspectos (cópia, reconhecimento de cores, entre outros) são passiveis de serem ensinados na primeira série do primeiro grau, estes últimos (operações cognitiva, por exemplo) são de mais difícil aquisição. Conforme Poppovic e col. (1975) " "1" assinalaram, "... operações cognitivas não podem ser adquiridas casualmente, ou num determinado momento que se resolva ensiná-las à criança... (elas) dependem de um longo processo evolutivo 
IFFRNANDES, J. \& GANDRA, Y.R. Avaliação inicial dos efeitos psico-pedagógicos dos Centros de Educação e Alimentação do Pré-Escolar. Rev. Saúde públ., S. Paulo, 15(supl.): 64-78, 1981.

e de um desenvolvimento harmonioso, constituido de etapas regidas por sequiências determinadas, em que cada nova aquisição baseia-se em fatores já existentes, assimilados por meio de maturidade, experiência e estimulação adequada".

As implicações educacionais dos resultados obtidos são bastante graves, uma vez que para as crianças de 6 anos aproxima-se o processo de escolarização formal no primeio grau, que pressupōe as habilidades e desempenhos que as crianças "Não-Ceapenses" apresentaram de modo limitado.

As diferenças observadas, em termos do total da "Escala de Comportamento", apontaram para a mesma direção. As avaliações do comportamento das crianças "Não-Ceapenses", durante a testagem, foram significativamente mais baixas que as das crianças "Ceapenses", em duas das três idades pesquisadas. Deve-se ressaltar que a única diferença năo significativa, aos 6 anos, provavelmente foi devida ao "efeito de limite" da própria "Escala", nesta idade.
Concluindo pode-se dizer que tanto uma avaliação fechada ("IADPE") como uma avaliação mais influenciada por fatores subjetivos ("Escala") revelaram diferenças significativas entre os grupos estudados e sugeriram a provável ocorrência de dificuldades no processo de escolarização das crianças que não frequientavam o programa.

Estudos posteriores devem permitir estimar o grau de generalidade destes resultados para CEAPEs de outras localidades, possibilitando conhecimento mais detalhado dos efeitos psico-pedagógicos do programa.

\section{AGRADECIMENTOS}

A equipe formada por psicólogos ou estudantes do Curso de Graduação do Instituto de Psicologia da Universidade de São Paulo: Aurora Coelho Pullin, Arlete Pogorelski, Eva Maria Migliavacca, Heloisa Lebrão, Hissako Fujihara, Lidia Yuri Kubota, Mario Sergio Picorelli, Norma Lottenberg, Maria José A.C. Veiga e Sonia Maria Pettincli.

FERNANDES, J. \& GANDRA, Y. R. LAn initial assessment of the psycho-pedagogical effects of the CEAPE program]. Rev. Saúde públ., S. Paulo, 15(suppl.):64-78, 1981.

ABSTRACT: The assessment of the socio-psycho-motor development of preschool children that participated in the CEAPE recreation program as compared to that of a control group of the same community, was carried out with the help of a set of tests prepared and pre-tested with that end in view. These preschoolers were also tested by the use of a behavior scale. In both areas of assessment CFAPE preschool children were seen to enjoy a considerable advantage over others. These results suggest that those children who do not attend CEAPE will probably face difficulties during their primary course.

UNITERMS: Preschool child, evaluation. Child development. CEAPE program, evaluation.

\section{REFERENCIAS BIBLIOGRAFICAS}

1. ANASTASI, A. Testes psicológicos. São Pa:-lo, Ed. Hernes, 1967.

2. CALDWELL, B.M. Cooperative preschool inventoru. Ed. rev. Princeton, N.J., Educational Testing Service, 1970.
3. CAVALCANTI, M.L.F, et al. Plano rotativo de recreação orientada. Bol. Ofic. OMEP, Rio de Janeiro, (3):5-10, maio/jun. 1976.

4. FERNANDES, J. \& GANDRA, Y.R. Instrumento de avaliação do desenvolvimento 
FERNANDES, J. \& GANDRA, Y.R. Avaliação inicial dos efeitos psico-pedagógicos dos Centros de Educação e Alimentação do Pré-Escolar. Rev. Saúde puibl., S. Paulo, 15(supl.): 64-78, 1981.

de pré-escolares dos Centros de Educação e Alimentação do Pré-Escolar. Rer. Saúde piubl., S. Paulo, 15(supl.): 79-90, 1981.

5. GANDRA, Y.R. Asistencia alimentaria por médio de centros de educación y alimentación del pre-escolar. Bot. Ofic. sanit. panamer, $74: 302-14,1973$.

6. GANDRA, Y.R. Nutrition and dietetic education program for preschool children at primary schools in Brazil. In: Anderson, M.A. \& Grewal. T., ed, Nutrition planning in developing world: proceed. ings of Regional Workshops held by CARE in India, Fenya and Colombia, 1976. Bogotá. Programas Editoriales. 1976. p. 205-12.

7 GOUVEIA. A.J. Professoras de amanhã: um estudo da escola ocupacional. São Paulo. Ed. Pioneira, 1970.
8. MACEDO, R.M.S. A organiząão grafo-perceptiva em pré-escolares de diferentes niveis sócio-econômico-educacionais. São Palilo, 1973. [Tese de Doutoramento Faculdade de Filosofia, Ciências e Letras de São Bento da PUC]

3. Poppovic, M.A. et al. Inventário de habilidades vara pré-escolares; Relatório pré-teste. São Paulo. Departamento de Pesquisas Etucacionais. Fundação Carlos Chagas, 1975.

10. POPPOVIC, M.A. et al. Marginalização cultural: subsidios para um currículo pre-escolar. Cad. Pesq. Fund. Carlos Chagas, 14:7-38,1975.

Recebido para publicasão em 10/0\%/1981

Aprovado para publicação em 17/11/1981 